\title{
Local Nash Inequality and Inhomogeneity of Heat Kernels
}

\author{
Jun Kigami \\ Graduate School of Informatics \\ Kyoto University \\ Kyoto 606-8501, Japan \\ e-mail:kigami@i.kyoto-u.ac.jp
}

\begin{abstract}
The local Nash inequality is introduced as a natural extension of the classical Nash inequality yielding space-homogeneous upper heat kernel estimate. The local Nash inequality contains local information of the heat kernel and is a necessary condition for the space-inhomogeneous heat kernel estimate involving volume of balls like the one obtained by Li-Yau[20] for a complete Riemannian manifold with non-negative Ricci curvature. Under the volume doubling property, the local Nash inequality combined with the exit time estimate is shown to be equivalent to a sub-Gaussian off-diagonal upper estimate of heat kernel allowing space-inhomogeneity
\end{abstract}

\section{Introduction}

In [21], Nash studied an asymptotic behavior of the fundamental solution of a parabolic partial differential equation. In a modern fashion, his result can be interpreted in the following way: let $(X, d)$ be a locally compact metric space and let $\mu$ be a Radon measure on $(X, d)$. Also let $-L$ be a generator of a strong continuous symmetric Markov semigroup $\left\{T_{t}\right\}_{t>0}$ on $L^{2}(X, \mu)$ and let $p(t, x, y)$ be the fundamental solution of $\partial u / \partial t=L u$, i.e., $\left(T_{t} u\right)(x)=\int_{X} p(t, x, y) u(y) \mu(d y)$. $p(t, x, y)$ is often called the heat kernel associated with $L$. If the Nash inequality

$$
\mathcal{E}(u, u)\|u\|_{1}^{4 / \theta} \geq c\|u\|_{2}^{2+4 / \theta}
$$

where $\mathcal{E}(u, u)=-(u, L u)$ and $\|\cdot\|_{p}$ is the $L^{p}$-norm, holds for any $u \in \operatorname{Dom}(\mathcal{E}) \cap$ $L^{1}$, then

$$
\sup _{x, y \in X} p(t, x, y) \leq c^{\prime} t^{-\theta / 2}
$$

for any $t>0$. In [5], the converse direction has been shown to be true as well. Nash's idea has been pursued by many authors, see $[25,6]$ for example, to replace the right-hand side of (1.2) by more general class of functions. Note that those upper estimates are homogeneous (i.e. uniform) with respect to the space $X$.

On the other hand, Li-Yau[20] showed that if $X$ is a complete Riemannian manifold with non-negative Ricci curvature, $d$ is the geodesic distance and $\mu$ is 
the Riemannian volume, then

$$
\frac{c_{1}}{V(\sqrt{t}, x)} \exp \left(-\frac{d(x, y)^{2}}{c_{2} t}\right) \leq p(t, x, y) \leq \frac{c_{3}}{V(\sqrt{t}, x)} \exp \left(-\frac{d(x, y)^{2}}{c_{4} t}\right)
$$

where $V(r, x)$ is the volume of $B_{r}(x)=\{y \mid d(x, y)<r\}$. For Riemannian manifolds, (1.3) is proven to be equivalent to the Poincare inequality and the volume doubling property: $V(2 r, x) \leq C V(r, x)$ for any $r>0$ and any $x \in X$, where $C$ is independent of $r$ and $x$. See [10] and [22]. Even as estimates of on-diagonal part $p(t, x, x),(1.3)$ has the great advantage in comparison with $(1.2)$, that is, (1.3) allows inhomogeneity with respect to the space $X$.

Recently, it has been shown in $[3,13]$ that certain heat kernels on fractals possesses strong space-inhomogeneity. Typical example is a time change of the Brownian motion on $[0,1]$ with respect to a self-similar measure $\mu$. If $\mu$ is not the Lebesgue measure, then $\overline{\lim }_{t \rightarrow 0}-\log p(t, x, x) / \log t$ is quite sensitive to $x$ and has multifractal structure. See Section 5 for details. Note that in the case of a Riemannian manifold, inhomogeneity as $t \rightarrow 0$ is much milder than such a situation because $V(\sqrt{t}, x)$ behaves like $t^{n / 2}$ as $t \rightarrow 0$.

The main purpose of this paper is to introduce a natural extension of the Nash inequality, called the local Nash inequality, which yield inhomogeneous (upper) heat kernel estimate as in (1.3) and can be applied to highly inhomogeneous cases like the ones on fractals. We say that the local Nash inequality holds if and only if there exists positive constants $A$ and $B$ such that

$$
\mathcal{E}(u, u)+A \frac{\|u\|_{1}^{2}}{r^{\beta} \inf _{y \in \operatorname{supp}(u)} V(r, y)} \geq B \frac{\|u\|_{2}^{2}}{r^{\beta}}
$$

for any $r>0$ and any $u \in \operatorname{Dom}(\mathcal{E}) \cap L^{1}$. As is seen in Section 3, if $\mu$ is Ahlfors regular with respect to the distance $d$, i.e.,

$$
b_{1} r^{\alpha} \leq V(r, x) \leq b_{2} r^{\alpha}
$$

for any $r>0$, where $\alpha$ is independent of $x$, then the local Nash inequality is equivalent to the classical Nash inequality (1.1). Before explaining what it is capable of, we review recent works on inhomogeneous estimate of heat kernels.

For random walks on infinite graphs, Grigori'yan-Telcs[12] has shown that the sub-Gaussian heat kernel estimate

$$
\begin{aligned}
\frac{c_{1}}{V\left(t^{1 / \beta}, x\right)} \exp \left(-\left(\frac{d(x, y)^{\beta}}{c_{2} t}\right)^{\frac{1}{\beta-1}}\right) & \leq p(t, x, y) \\
& \leq \frac{c_{3}}{V\left(t^{1 / \beta}, x\right)} \exp \left(-\left(\frac{d(x, y)^{\beta}}{c_{4} t}\right)^{\frac{1}{\beta-1}}\right)
\end{aligned}
$$

where $d(x, y)$ is the minimal number of steps between $x$ and $y$, is equivalent to the volume doubling property + the Harnack inequality + the exit time estimate:

$$
a_{1} r^{\beta} \leq E_{x}\left(\tau_{B_{r}(x)}\right) \leq a_{2} r^{\beta}
$$


where $\tau_{B_{r}(x)}$ is the exit time from $B_{r}(x)$ and $E_{x}$ is the expectation with respect to the process $\left(\left\{X_{t}\right\}_{t>0},\left\{P_{x}\right\}_{x \in X}\right)$ associated with the heat kernel. They introduced a new method by proving a kind of maximum principle for polyharmonic functions as a substitute of Nash inequality in homogeneous estimates. This method has been used in $[23,24]$ to generalize results in [12].

The sub-Gaussian estimate which introduces the exponent $\beta$ in (1.6) originates in the study of diffusion processes on self-similar sets. Barlow-Perkins [4] proved that the heat kernel associated with the Brownian motion on the Sierpinski gasket satisfies a space homogeneous version of (1.6), where $V(r, x)=r^{\alpha}$, $\alpha$ is the Hausdorff dimension and $\beta$ is the walk dimension. The same estimate has been found true for Nested fractals in [19], affine nested fractals in [8] and Sierpinski carpets in [2].

Note that the distances $d$ are geodesic distances in both cases of (1.3) and (1.6). In general, we can not expect upper and lower off-diagonal estimates (1.6) for diffusions on metric spaces without geodesic distances. In fact, HamblyKumagai[14] found diffusion processes on self-similar sets with the volume doubling property, the Harnack inequality and the exit time estimate which do not satisfy the off-diagonal lower estimate in (1.6). To include such examples, we will focus on on-diagonal upper estimate

$$
p(t, x, x) \leq \frac{c}{V\left(t^{1 / \beta}, x\right)},
$$

on-diagonal lower estimate

$$
\frac{c}{V\left(t^{1 / \beta}, x\right)} \leq p(t, x, x)
$$

and off-diagonal upper estimate

$$
p(t, x, y) \leq \frac{c_{3}}{V\left(t^{1 / \beta}, x\right)} \exp \left(-\left(\frac{d(x, y)^{\beta}}{c_{4} t}\right)^{\frac{1}{\beta-1}}\right) .
$$

The main point of this paper is to show the following two facts:

(I) The on-diagonal upper estimate (1.8) implies the local Nash inequality (1.4).

(II) Under the volume doubling property, the local Nash inequality (1.4) and the exit time estimate (1.7) is equivalent to the off-diagonal upper estimate (1.10).

Moreover, under the volume doubling property, the on-diagonal lower estimate (1.9) is derived from the exit time estimate (1.7) by a modification of existing methods in $[1,3,8,11]$. Hence as for on-diagonal estimates, we have a complete picture under the volume doubling condition.

Unlike the classical case, the local Nash inequality alone does not seem to give the upper on-diagonal estimate. It leads us to an on-diagonal estimate of heat kernel with Dirichlet 0-boundary condition on the boundary of a ball. More precisely, the local Nash inequality and the volume doubling property suffice an estimate, $p^{r, x}\left(r^{\beta}, x, x\right) \leq c V(r, x)^{-1}$, where $p^{r, x}(t, z, y)$ is the heat kernel 
associated with the process killed at the boundary of $B_{r}(x)$. See Lemma 4.3 for details. We need the exit time estimate to fill the gap between the local Dirichlet kernel $p^{r, x}$ and the global kernel $p$. At this point, we do not know that this is technical problem or not.

This method can be applied to the highly inhomogeneous heat kernels on fractals, for example, the Sierpinski gasket and the (higher-dimensional) Sierpinski carpet. We will do this in subsequent paper [16]. In this paper, we will present a special example, a time change of the Brownian motion on $[0,1]$ with respect to a self-similar measure. If the self-similar measure has the volume doubling property with respect to the Euclidean distance, then there exist $\beta>0$ and a distance $d$ on $[0,1]$ under which the exit time estimate and the local Nash inequality holds. Moreover, in this case, $d$ is a geodesic distance and we will show lower and upper off-diagonal estimate (1.6). See Section 5 for details.

The organization of this paper is as follows. In Section 2, we state the main results including (I) and (II) above. In Section 3, we will study the case where $V(r, x)$ does not depend on $x$ and show that the local Nash inequality is the natural extension of the classical one. In Section 4, we will give proofs to the main results. Section 5 is devoted to an example which we mentioned above.

The author wish to express his gratitude to Professors T. Kumagai and M. Hino for stimulating discussions and fruitful comments.

\section{Main results}

In this section, we will state the main results of this paper. We start with clarifying our setting. In this paper, $(X, d)$ is a locally compact and connected metric space. $\mu$ is a Radon measure on $(X, d)$ satisfying that $\mu(O)>0$ for any non-empty open set $O \subseteq X$. $(\mathcal{E}, \mathcal{F})$ is a local regular Dirichlet form on $L^{2}(X, \mu)$ and $\left\{T_{t}\right\}_{t>0}$ is the strongly continuous semigroup associated with the Dirichlet form $(\mathcal{E}, \mathcal{F})$ on $L^{2}(X, \mu)$. We also assume that the semigroup $\left\{T_{t}\right\}_{t>0}$ is ultracontractive, i.e. $T_{t}$ can be extended to an bounded operator from $L^{2}(X, \mu)$ to $L^{\infty}(X, \mu)$. Then, $T_{t}$ has an symmetric integral kernel $p(t, x, y)$ for any $t>0$ that belongs to $L^{\infty}\left(X^{2}, \mu \times \mu\right)$. See [7, Lemma 2.1.2] for example. We call $p(t, x, y)$ the heat kernel associated with $\left\{T_{t}\right\}_{t>0}$. Also we use $\left(\left\{X_{t}\right\}_{t>0},\left\{P_{x}\right\}_{x \in X}\right)$ to denote the diffusion process on $(X, d)$ associated with the local regular Dirichlet form $(\mathcal{E}, \mathcal{F})$ on $L^{2}(X, \mu) . E_{x}(\cdot)$ is the expectation with respect to $P_{x}$.

Notation. We write $B_{r}(x)=\{y \mid y \in X, d(x, y)<r\}$. and $V(r, x)=\mu\left(B_{r}(x)\right)$. We also use $\mathbb{R}_{+}^{*}=(0,+\infty) \cup\{+\infty\} .\left(0, r_{*}\right]$ means $(0,+\infty)$ when $r_{*}=+\infty$.

First we show an inequality regarding Dirichlet forms.

Theorem 2.1. Let $u \in \mathcal{F} \cap L^{1}(X, \mu)$. Then, for any $t>0$,

$$
\mathcal{E}(u, u)+t^{-1}\|u\|_{1}^{2} \operatorname{esssup}_{x, y \in \operatorname{supp}(u)} p(t, x, y) \geq t^{-1}\|u\|_{2}^{2},
$$


where ess.sup is the essential supremum and $\|\cdot\|_{p}$ is the $L^{p}$-norm with respect to the measure $\mu$. In particular,

$$
\mathcal{E}(u, u)+t^{-1}\|u\|_{1}^{2}\left\|T_{t}\right\|_{1 \rightarrow \infty} \geq t^{-1}\|u\|_{2}^{2}
$$

for any $u \in \mathcal{F} \cap L^{1}(X, \mu)$, where $\|\cdot\|_{p \rightarrow q}$ is the operator norm of an bounded operator from $L^{p}(X, \mu)$ to $L^{q}(X, \mu)$.

This inequality (2.1) is a connection between the local Nash inequality defined below and heat kernel estimates.

To show Theorem 2.1, we need the next lemma which is verified easily by using the spectral representation of $\left\{T_{t}\right\}_{t>0}$ and its generator.

Lemma 2.2. For any $t>0$ and any $u \in L^{2}(X, \mu)$, define

$$
\widetilde{\mathcal{E}}_{t}(u)=t^{-1}\left(\|u\|_{2}^{2}-\int_{X \times X} p(t, x, y) u(x) u(y) \mu(d x) \mu(d y)\right) .
$$

Then $\widetilde{\mathcal{E}}_{t}(u)$ is monotonically decreasing and $u \in \mathcal{F}$ if and only if $\lim _{t \downarrow 0} \widetilde{\mathcal{E}}_{t}(u)<$ $+\infty$. Moreover, if $u \in \mathcal{F}$, then $\lim _{t \downarrow 0} \widetilde{\mathcal{E}}_{t}(u)=\mathcal{E}(u, u)$.

Proof of Theorem 2.1. Since

$$
\int_{X \times X} p(t, x, y) u(x) u(y) \mu(d x) \mu(d y) \leq \operatorname{ess.sup}_{x, y \in \operatorname{supp}(u)} p(t, x, y)\|u\|_{1}^{2} .
$$

Lemma 2.2 implies

$$
\mathcal{E}(u, u) \geq \widetilde{\mathcal{E}}_{t}(u) \geq t^{-1}\left(\|u\|_{2}^{2}-\operatorname{ess.sup}_{x, y \in \operatorname{supp}(u)} p(t, x, y)\|u\|_{1}^{2}\right) .
$$

Now we define the local Nash inequality.

Definition 2.3 (Local Nash inequality). Let $\beta>0, r_{1} \in \mathbb{R}_{+}^{*}$ and $r_{2} \in \mathbb{R}_{+}^{*}$ and let $h:\left(0, r_{1}\right] \times X \rightarrow(0,+\infty)$. We say that the local Nash inequality with indices $\left(h, \beta, r_{1}, r_{2}\right)$ holds if and only if there exists positive constants $A$ and $B$ such that

$$
\mathcal{E}(u, u)+A \frac{\|u\|_{1}^{2}}{r^{\beta} \inf _{y \in \operatorname{supp}(u)} h(r, y)} \geq B \frac{\|u\|_{2}^{2}}{r^{\beta}}
$$

for any $r \in\left(0, r_{1}\right]$ and any $u \in \mathcal{F} \cap L^{1}(X, \mu)$ with $\operatorname{diam}(\operatorname{supp}(u)) \leq r_{2}$.

In particular, if $h(r, x)=H(r)$ for some $H:\left(0, r_{1}\right] \rightarrow(0,+\infty)$, then (2.4) is

$$
\mathcal{E}(u, u)+A \frac{\|u\|_{1}^{2}}{r^{\beta} H(r)} \geq B \frac{\|u\|_{2}^{2}}{r^{\beta}} .
$$

We call this the homogeneous Nash inequality. In Section 3, the homogeneous Nash inequality is shown to be equivalent to the heat kernel estimate $p(t, x, y) \leq c H\left(t^{1 / \beta}\right)^{-1}$ and, if $H(r)=r^{\alpha}$, it is also equivalent to the classical Nash Inequality.

Immediately by the above theorem, an upper estimate of heat kernel implies the local Nash inequity as follows. 
Corollary 2.4. Let $t_{*} \in \mathbb{R}_{+}^{*}$ and let $h:\left(0, t_{*}\right] \times X \rightarrow(0,+\infty)$ If there exists $c>0$ such that

$$
p(t, x, x) \leq c \frac{1}{h\left(t^{1 / \beta}, x\right)}
$$

for any $t \in\left(0, t_{*}\right]$ and any $x \in X$, then the local Nash inequality with indices $\left(h, \beta, r_{*},+\infty\right)$ holds, where $r_{*}=\left(t_{*}\right)^{1 / \beta}$.

Proof. Since

$$
p(t, x, y)=\int_{X} p(t / 2, x, z) p(t / 2, y, z) \mu(d z),
$$

the Schwartz inequality implies that $p(t, x, y)^{2} \leq p(t, x, x) p(t, y, y)$. Hence by (2.1) and (2.6), we have the local Nash inequality.

Next we present results in the opposite direction. Namely the local Nash inequality together with the volume doubling property and the exit time estimate implies an inhomogeneous heat kernel estimate.

Definition 2.5. Let $r_{*} \in \mathbb{R}_{+}^{*} \cdot \mu$ is said to have the volume doubling property with respect to the distance $d$ on $\left(0, r_{*}\right]$ if and only if there exists $c>1$ and $\gamma \in(0,1)$ such that $V(r, x) \leq c V(\gamma r, x)$ for any $x \in X$ and any $r \in\left(0, r_{*}\right]$.

As a generalization of $V(r, x)$ where $\mu$ has the volume doubling property, we define the notion of a doubling gauge as follows.

Definition 2.6. Let $r_{*} \in \mathbb{R}_{+}^{*}$. A function $h:\left(0, r_{*}\right] \times X \rightarrow(0,+\infty)$ is called a doubling gauge on $\left(0, r_{*}\right]$ if and only if the following four conditions are satisfied: (H1) For any $x \in X, h(r, x)$ is monotonically increasing with respect to $r$.

(H2) There exists $c>1$ and $\gamma \in(0,1)$ such that $h(r, x) \leq \operatorname{ch}(\gamma r, x)$ for any $(r, x) \in\left(0, r_{*}\right] \times X$.

(H3) There exists $M>0$ such that $h(r, x) \leq M h(r, y)$ for any $r \in\left(0, r_{*}\right]$ and any $x, y \in X$ with $d(x, y) \leq r$.

(H4) There exists $r^{\prime} \in\left(0, r_{*}\right]$ such that $\inf _{x \in X} h\left(r^{\prime}, x\right)>0$.

Lemma 2.7. If $\mu$ has the volume doubling property on $\left(0, r_{*}\right]$ and there exists $r^{\prime} \in\left(0, r_{*}\right]$ such that in $f_{x \in X} V\left(r^{\prime}, x\right)>0$, then $V(r, x)$ is a doubling gauge on $\left(0, r_{*} / 2\right]$. In particular, if $(X, d)$ is bounded and $\mu$ has the volume doubling property on $(0, \operatorname{diam}(X)]$, then $V(r, x)$ is a doubling gauge on $(0,+\infty)$.

Proof. To prove the first part of the statement, we need to show (H3). Let $r \in\left(0, r_{*} / 2\right]$ and let $d(x, y) \leq r$. Then $B_{r}(y) \subseteq B_{2 r}(x)$. By the volume doubling property, $V(2 r, x) \leq M V(r, x)$ for any $r \in\left(0, r_{*} / 2\right]$ and any $x \in X$, where $M$ is independent of $r$ and $x$. Hence $V(r, y) \leq M V(r, x)$. This shows (H3). Next assume that $(X, d)$ is bounded. Note that if the volume doubling property holds on $(0, \operatorname{diam}(X)]$, then it holds on $(0,+\infty)$ as well. Hence we have (H3) for any $r>0$. (H4) is trivial because $B_{r}(x)=B_{r}(y)=X$ if $r \geq \operatorname{diam}(X)$. 
Definition 2.8. Let $r_{*} \in \mathbb{R}_{+}^{*}$. We say the exit time estimate with indices $\left(\beta, r_{*}\right)$ holds if there exist positive constants $a_{1}$ and $a_{2}$ such that

$$
a_{1} r^{\beta} \leq E_{x}\left(\tau_{B_{r}(x)}\right) \leq a_{2} r^{\beta}
$$

for any $r \in\left(0, r_{*}\right]$ and any $x \in X$, where $\tau_{A}$ is the exit time from $A \subset X$ defined by $\tau_{A}=\inf \left\{t \mid X_{t} \notin A\right\}$.

Remark. If $(X, d)$ is bounded, then $r_{*}$ can not be $+\infty$. Note that $\tau_{B_{r}(x)}=+\infty$ for $r>\operatorname{diam}(X)$.

The local Nash inequality together with the exit time estimate suffices for an off-diagonal upper heat kernel estimate as follows.

Theorem 2.9. Let $\beta>0$. Let $r_{1}$ and $r_{2}$ belong to $\mathbb{R}_{+}^{*}$ and let $h$ be a doubling gauge on $\left(0, r_{1}\right]$. Suppose that $p(t, x, y)$ is jointly continuous on $(0,+\infty) \times X \times X$. Also assume the following two conditions:

(A1) The local Nash inequality with indices $\left(h, \beta, r_{1},+\infty\right)$ holds.

(A2) The exit time estimate with indices $\left(\beta, r_{2}\right)$ holds

Then, $\beta>1$ and there exist positive constants $c_{1}, c_{2}, r_{*}$ and $t_{*}$ such that

$$
p(t, x, y) \leq \frac{c_{1}}{h\left(t^{1 / \beta}, x\right)} \exp \left(-c_{2}\left(\frac{d(x, y)^{\beta}}{t}\right)^{\frac{1}{\beta-1}}\right)
$$

for any $x, y \in X$ with $d(x, y) \leq r_{*}$ and any $t \in\left(0, t_{*}\right]$. Moreover, if $r_{1}=+\infty$, then $t_{*}=+\infty$ and if $r_{2}=+\infty$ or $(X, d)$ is bounded, then $r_{*}=+\infty$.

We give a proof of this theorem in Section 4 .

Remark. (1) The conditions (A1) and (H4) can be replaces by

(B1) The local Nash inequality with indices $\left(h, \beta, r_{1}, r_{3}\right)$ holds for some $r_{3}>0$ and

(B2) There exist positive constants $c>0$ and $\lambda>0$ such that $\sup _{x \in X} p(t, x, x) \leq$ $c t^{-\lambda}$ for any $t \in(0,1)$.

(2) If $(X, d)$ is bounded then (2.8) with a finite $r_{*}$ implies (2.8) with $r_{*}=+\infty$. Furthermore, if $h$ is a doubling gauge on $(0, \operatorname{diam}(X)]$, then $t_{*}=+\infty$.

Next result gives a converse of the above theorem in case $h(r, x)=V(r, x)$. It shows that the upper heat kernel estimate $(2.8)$ is equivalent to $(\mathrm{A} 1)+(\mathrm{A} 2)$. Note that by Corollary 2.4, (2.8) implies the local Nash inequality.

Theorem 2.10. Assume that $(\mathcal{E}, \mathcal{F})$ is conservative, i.e. $1 \in \mathcal{F}$ and $\mathcal{E}(1,1)=0$, and that $\mu$ has the volume doubling property on $(0,+\infty)$. Let $\beta>1$. If there exist positive constants $c_{1}, c_{2}$ and $t_{*} \in \mathbb{R}_{+}^{*}$ such that

$$
p(t, x, y) \leq \frac{c_{1}}{V\left(t^{1 / \beta}, x\right)} \exp \left(-c_{2}\left(\frac{d(x, y)^{\beta}}{t}\right)^{\frac{1}{\beta-1}}\right)
$$

for any $x, y \in X$ and any $t \in\left(0, t_{*}\right]$, then the exit time estimate with indices $\left(\beta, r_{*}\right)$ holds for some $r_{*} \in \mathbb{R}_{+}^{*}$. Moreover, if $t_{*}=+\infty$ and $\operatorname{diam}(X)=+\infty$, then $r_{*}=+\infty$. 
We will prove this theorem in Section 4 .

If $(X, d)$ is bounded, then the equivalence between the local Nash inequality + the exit time estimate and (2.9) can be stated in the following way.

Corollary 2.11. Suppose that $(X, d)$ is bounded. Assuem that $(\mathcal{E}, \mathcal{F})$ is conservative and that $\mu$ has the volume doubling property on $(0, \operatorname{diam}(X)]$. Let $\beta>1$. Then the following conditions $(\mathrm{C} 1)$ and $(\mathrm{C} 2)$ are equivalent.

(C1) The local Nash inequality with indices $\left(V, \beta, r_{0},+\infty\right)$ holds for some $r_{0} \in$ $\mathbb{R}_{+}^{*}$ and the exit time estimate with indices $\left(\beta, r_{1}\right)$ holds for some $r_{1}>0$.

(C2) There exist positive constants $c_{1}$ and $c_{2}$ such that (2.9) holds for any $x, y \in X$ and any $t>0$.

Proof. By Lemma 2.7, $V(r, x)$ is a doubling gauge. Hence by Theorem 2.9, $(\mathrm{C} 1)$ implies (C1). Also by Corollary 2.4 and Theorem 2.10, (C2) implies (C1).

Finally we present results on the lower estimate of the heat kernel. To obtain the on-diagonal estimate, what we need is the exit time estimate and the volume doubling property. For off-diagonal estimate, we require the following properties.

Definition 2.12. (1) The distance $d$ on $X$ is said to have the midpoint property if and only if there exists $z \in X$ such that $d(x, z)=d(z, y)=d(x, y) / 2$ for any $x, y \in X$.

(2) Let $t_{*} \in \mathbb{R}_{+}^{*}$. We say that near diagonal estimate on $\left(0, t_{*}\right]$ holds if there exist positive constants $c$ and $\epsilon$ such that

$$
\frac{c}{V\left(t^{1 / \beta}, x\right)} \leq p(t, x, y)
$$

for any $t \in\left(0, t_{*}\right]$ and any $x, y \in X$ with $d(x, y) \leq \epsilon t^{1 / \beta}$.

If the distance $d$ has the midpoint property, there exists a geodesic between any two points $x$ and $y$ : there exists a continuous function $g:[0, d(x, y)] \rightarrow X$ such that $g(0)=x, g(1)=y$ and $d(g(t), g(s))=|t-s|$ for any $t, s \in[0, d(x, y)]$. See [1, Lemma 3.1]. A distance with the midpoint property is often called a geodesic distance.

If $\mu$ is Ahlfors regular with respect to $d$, i.e. (1.5) holds, then there is a established technique to obtain on and off-diagonal lower estimate of heat kernels. See $[1,3,8,11]$ for example. Making necessary adjustments to the techniques in those papers, we can obtain the following theorem.

Theorem 2.13. Let $r_{*} \in \mathbb{R}_{+}^{*}$. Suppse that $(\mathcal{E}, \mathcal{F})$ is conservative. Assume the exit time estimate with indices $\left(\beta, r_{*}\right.$ and that $\mu$ has the volume doubling property on $\left(0, r_{*}\right]$. Then $\beta>1$ and there exist positive constants $c$ and $t_{*}$ such that

$$
\frac{c}{V\left(t^{1 / \beta}, x\right)} \leq p(t, x, x)
$$


for any $x \in X$ and any $t \in\left(0, t_{*}\right]$. Moreover if $r_{*}=+\infty$ or $(X, d)$ is bounded, then $t_{*}=+\infty$. Furthermore, if we assume, in addition, the midpoint property of $d$ and the near diagonal lower estimate on $\left(0, t_{*}\right]$, then there exist positive constants $c_{1}$ and $c_{2}$ such that

$$
\frac{c_{1}}{V\left(t^{1 / \beta}, x\right)} \exp \left(-c_{2}\left(\frac{d(x, y)^{\beta}}{t}\right)^{\frac{1}{\beta-1}}\right) \leq p(t, x, y)
$$

for any $x, y \in X$ and any $t \in\left(0, t_{*}\right]$.

By Theorems 2.10 and 2.13, it follows that the off-diagonal upper estimate (2.9) implies the diagonal lower estimate (2.11) under the hypothesis of Theorem 2.10 .

\section{Homogeneous case}

In this section, we consider homogeneous cases to illustrate that the local Nash Inequality is a natural extension of the classical Nash inequality.

Through this section, $Y$ is a locally compact metric space and $\nu$ is a Radon measure on $Y$ which satisfies that $\nu(O)>0$ for any non-empty open set $O \subset Y$. $(\mathcal{E}, \mathcal{F})$ is a Dirichlet form on $L^{2}(Y, \nu)$ and $\left\{T_{t}\right\}_{t>0}$ be the associated semigroup on $L^{2}(Y, \nu)$.

The next lemma is obtained essentially by the classical argument from the Nash inequality to the heat kernel estimate.

Lemma 3.1. Let $A, B, \alpha, \beta$ and $R$ be positive numbers. If

$$
\mathcal{E}(u, u)+\frac{A\|u\|_{1}^{2}}{r^{\alpha+\beta}} \geq \frac{B\|u\|_{2}^{2}}{r^{\beta}}
$$

for any $u \in \mathcal{F} \cap L^{1}(Y, \nu)$ and any $r \in(0, R]$, then $T_{t}\left(L^{1}(Y, \nu)\right) \subseteq L^{\infty}(Y, \nu)$ and

$$
\left\|T_{\eta R^{\beta}}\right\|_{1 \rightarrow \infty} \leq A B^{-1} R^{-\alpha} \max \left\{c_{1}, B^{-\alpha / \beta} c_{2} \eta^{-\alpha / \beta}\right\}
$$

for any $\eta>0$, where $c_{1}$ and $c_{2}$ are positive constants which only depends on $\alpha$ and $\beta$.

Proof. Renormalizing the parameters as $\tilde{r}=r^{\beta}$ and $\tilde{\alpha}=\alpha / \beta$, we may assume that $\beta=1$ without loss of generality. Set $a=A\|u\|_{1}^{2}$ and $b=B\|u\|_{2}^{2}$. Define $f(x)=b x-a x^{1+\alpha}$ and let $M=\max _{x \in[1 / R,+\infty)} f(x)$. Let $x_{*}=(1+$ $\alpha)^{-1 / \alpha}(a / b)^{1 / \alpha}$. Then $M=f(1 / R)$ if $x_{*} \leq 1 / R$ and $M=\gamma b^{1+1 / \alpha} a^{-1 / \alpha}$ if $x_{*} \geq 1 / R$, where $\gamma=(1+\alpha)^{-1 / \alpha}-(1+\alpha)^{-1-1 / \alpha}$. This implies either

$$
\mathcal{E}(u, u) A^{1 / \alpha}\|u\|_{1}^{2 / \alpha} \geq \gamma B^{1+1 / \alpha}\|u\|_{2}^{2+2 / \alpha} \quad \text { or } \quad R^{\alpha} B\|u\|_{2}^{2} \leq A\|u\|_{1}^{2} .
$$

Now let $u \in L^{2}(Y, \nu) \cap L^{1}(Y, \nu)$ with $\|u\|_{1}=1$ and define $g(t)=\left\|T_{t} u\right\|_{2}^{2}$. Since $T_{t} u \in L^{1}(Y, \nu)$ and $\left\|T_{t} u\right\|_{1} \leq\|u\|_{1}$, we see that $\left\|T_{t} u\right\|_{1} \leq 1$. Also $g^{\prime}(t)=$ $-2 \mathcal{E}\left(T_{t} u, T_{t} u\right)$. Hence by $(3.3)$,

$$
\left(g(t)^{-1 / a}\right)^{\prime} \geq 2 \gamma \alpha^{-1} A^{-1 / \alpha} B^{1+1 / \alpha} \quad \text { or } \quad g(t) \leq A B^{-1}(1+\alpha) R^{-\alpha} .
$$


Solving the differential inequality, we obtain

$$
g(\eta R) \leq A B^{-1} R^{-\alpha} \max \left\{1+\alpha,(\alpha / 2 \gamma)^{\alpha} B^{-\alpha} \eta^{-\alpha}\right\} .
$$

Therefore, $\left\|T_{\eta R}\right\|_{1 \rightarrow 2}$ is bounded by the square root of the right hand side of the above inequity. By using the duality, $\left\|T_{t}\right\|_{2 \rightarrow \infty}=\left\|T_{t}\right\|_{1 \rightarrow 2}$. Since $\left\|T_{t}\right\|_{1 \rightarrow \infty} \leq$ $\left\|T_{t / 2}\right\|_{1 \rightarrow 2}|| T_{t / 2} \|_{2 \rightarrow \infty}$, we obtain the desired inequality.

As in the classical case, the homogeneous Nash inequalities are equivalent to certain estimate of heat kernels.

Theorem 3.2. Let $\beta>0$ and let $H:\left(0, r_{*}\right] \rightarrow(0,+\infty)$ for some $r_{*} \in \mathbb{R}_{+}^{*}$. Assume that $H$ is monotonically increasing and satisfies the doubling condition (H2) with $h(r, x)=H(r)$. Then the following two conditions are equivalent.

(1) There exists $c>0$ such that $\left\|T_{t}\right\|_{1 \rightarrow \infty} \leq c H\left(t^{1 / \beta}\right)^{-1}$ for any $t \in\left(0,\left(r_{*}\right)^{\beta}\right]$.

(2) The homogeneous Nash inequality with indices $\left(H, r_{*}\right)$ holds, i.e. (2.5) is satisfied for any $r \in\left(0, r_{*}\right]$ and any $u \in \mathcal{F} \cap L^{1}(Y, \nu)$.

Furthermore, if $H(r)=r^{\alpha}$ for some $\alpha>0$, the following condition (3) is also equivalent to (1) and (2).

(3) There exist positive constants $c$ and $C$ such that

$$
\left(c \mathcal{E}(u, u)+\delta\|u\|_{2}^{2}\right)\|u\|_{1}^{2 \beta / \alpha} \geq C\|u\|_{2}^{2+2 \beta / \alpha}
$$

for any $u \in \mathcal{F} \cap L^{1}(Y, \nu)$, where $\delta=1$ if $r_{*}<+\infty$ and $\delta=0$ if $r_{*}=+\infty$.

Note that (3.4) is the classical Nash inequality mentioned in the introduction.

In $[25,6]$, different type of extension of the classical Nash inequality is proven to be equivalent to the homogeneous upper estimate of a heat kernel. In short, their result is that

$$
\theta\left(\|u\|_{2}^{2}\right) \leq \mathcal{E}(u, u)
$$

for any $u \in \mathcal{F} \cap L^{1}(Y, \nu)$ with $\|u\|_{1}=1$, where $\theta$ satisfies $-m^{\prime}(t)=\theta(m(t))$ is equivalent to $\left\|T_{t}\right\|_{1 \rightarrow \infty} \leq m(t)$. Our result shows that (3.5) is equivalent to the homogeneous Nash inequality with $H\left(t^{1 / \beta}\right)^{-1}=m(t)$ if $H$ has the volume doubling property. Note that Coulhon's result in [6] can be applied even when $H$ does not possess the volume doubling property.

Proof. (1) $\Rightarrow(2)$ : Letting $t=r^{\beta}$ in (2.2), we immediately see (3).

$(2) \Rightarrow(1)$ : Since $H$ has the doubling property, there exist positive constants $\lambda$ and $c_{0}$ such that $H(r) \geq c_{0}(r / R)^{\lambda} H(R)$ for any $R \in\left(0, r_{*}\right]$, any $r \in(0, R]$. (See Lemma 4.1.) Therefore, for any $R \in\left(0, r_{*}\right]$,

$$
\mathcal{E}(u, u)+\frac{A R^{\lambda}\|u\|_{1}^{2}}{c_{0} H(r) r^{\beta+\lambda}} \geq \frac{B\|u\|_{2}^{2}}{r^{\beta}}
$$

Letting $\eta=1$ in Lemma 3.1, we have $\left\|T_{R^{\beta}}\right\|_{1 \rightarrow \infty} \leq c H(R)^{-1}$, where $c$ is independent of $R$.

$(1) \Leftrightarrow(3)$ : Since (3.4) is the Nash inequality, this is the classical result. See [5] and [17, Appendix B.3] for example. 


\section{Proof of main results}

In this section, we will give proofs of the results in Section 2.

First we give a equivalent condition for the volume doubling property.

Lemma 4.1. Let $h$ be a doubling gauge on $\left(0, r_{*}\right]$. Then there exist positive constants $\lambda$ and $c_{0}$ such that $h(r, x) \geq c_{0}(r / R)^{\lambda} h(R, x)$ for any $R \in\left(0, r_{*}\right]$, any $r \in(0, R]$ and any $x \in X$. Moreover, there exists $C>0$ such that $h(r, x) \geq$ $C\left(r / r_{*}\right)^{\lambda}$ for any $r \in\left(0, r_{*}\right]$.

Proof. By (H2), for any $n \geq 0, h(R, x) \leq c^{n} h\left(\gamma^{n} R, x\right)$. Therefore, if $\gamma^{n} R \geq r \geq$ $\gamma^{n+1} R$, we have $h(R, x) \leq c^{n} h(r, x)$. This immediately imply the lemma.

Next lemma gives a homogeneous bound of the heat kernel.

Lemma 4.2. Let $h$ be a doubling gauge. Under the condition (A1), there exist positive constants $\gamma_{1}$ and $\gamma_{2}$ such that $\left\|T_{t}\right\|_{1 \rightarrow \infty} \leq \max \left\{\gamma_{1}, \gamma_{2} t^{-\lambda / \beta}\right\}$ for any $t>0$, where $\lambda$ is the constant appeared in Lemma 4.1.

Proof. Lemma 4.1 along with the local Nash inequality implies (3.1) with $\alpha=\lambda$. Hence by Lemma 3.1, we have the desired estimate.

We now try to extract local behavior of the heat kernel from the local Nash inequality. Let $U$ be an open set. Define $\mathcal{F}_{U}=\{u \mid u \in \mathcal{F}, \operatorname{supp}(u) \subseteq U\}$ and $\mathcal{E}_{U}=\left.\mathcal{E}\right|_{\mathcal{F}_{U} \times \mathcal{F}_{U}}$. Also $\mu_{U}$ is the restriction of $\mu$ on $U$. Then by [9, Theorem 4.4.3], $\left(\mathcal{E}_{U}, \mathcal{F}_{U}\right)$ is a local regular Dirichlet form and $P_{x}^{U}\left(X_{t}^{U} \in A\right)=P_{x}\left(X_{t} \in A, \tau_{U} \geq\right.$ $t)$, where $\left(\left\{X_{t}^{U}\right\}_{t>0},\left\{P_{x}^{U}\right\}_{x \in U}\right)$ is the diffusion process associated with $\left(\mathcal{E}_{U}, \mathcal{F}_{U}\right)$ on $L^{2}\left(U, \mu_{U}\right)$. The semigroup associated with $\left(\mathcal{E}_{U}, \mathcal{F}_{U}\right)$ is denoted by $\left\{T_{t}^{U}\right\}_{t>0}$. In case $U=B_{r}(x)$, we write $T_{t}^{r, x}$ in place of $T_{t}^{U}$ and use $p^{r, x}(t, y, z)$ to denote the associated heat kernel.

Lemma 4.3. Let $h$ be a doubling gauge on $\left(0, r_{*}\right]$. If the local Nash inequality with indices $\left(h, \beta, r_{*}, r_{*}\right)$ holds, then there exist positive constants $b_{1}$ and $b_{2}$ such that

$$
\left\|T_{\eta R^{\beta}}^{R, x}\right\|_{1 \rightarrow \infty} \leq \frac{1}{h(R, x)} \max \left\{b_{1}, b_{2} \eta^{-\lambda / \beta}\right\}
$$

for any $R \in\left(0, r_{*}\right]$, any $\eta>0$ and any $x \in X$.

Proof. By Lemma 4.1, $h(r, y) \leq c_{0}(r / R)^{\lambda} h(R, y)$. The property (H3) implies that $h(r, y) \leq c_{0} M(r / R)^{\lambda} h(R, x)$ for any $y \in B_{R}(x)$. Hence by the local Nash inequality,

$$
\mathcal{E}(u, u)+\frac{c_{0} M R^{\lambda}\|u\|_{1}^{2}}{h(R, x) r^{\beta+\lambda}} \geq \frac{c^{\prime}\|u\|_{2}^{2}}{r^{\beta}} .
$$

for any $r \in(0, R]$ and any $u \in \mathcal{F}_{B_{R}(x)}$. Applying Lemma 3.1 to $\left(\mathcal{E}_{B_{R}(x)}, \mathcal{F}_{B_{R}(x)}\right)$, we obtain (4.1).

We obtain the next lemma by following the discussions from Lemma 3.16 to the proof of Theorem 3.11 in [1]. 
Lemma 4.4. Assume the exit time estimate with indices $\left(\beta, r_{0}\right)$. Then $\beta>1$ and there exists positive constants $c, c^{\prime}$ and $r_{*}$ such that

$$
P_{x}(\tau(r, x) \leq t) \leq c \exp \left(-c^{\prime}\left(\frac{r^{\beta}}{t}\right)^{\frac{1}{\beta-1}}\right)
$$

for any $x \in X$, any $t>0$ and any $r \in\left(0, r_{*}\right]$, where $\tau(r, x)=\tau_{B_{r}(x)}$. Moreover if $r_{0}=+\infty$ or $(X, d)$ is bounded, then $r_{*}=+\infty$.

The following fact is essentially shown in the proof of Theorem 3.4 of [3].

Lemma 4.5. If $p(t, x, y)$ is jointly continuous, then

$$
p(t, x, x) \leq\left\|T_{t}^{R, x}\right\|_{1 \rightarrow \infty}+2 \sup _{y \in B_{r}(x)} P_{y}(\tau(x, R) \leq 2 / t) \sup _{z \in D_{R}(x)} p(t / 2, z, z)
$$

for any $R>0$, any $r \in(0, R]$ and any $x \in X$, where $D_{R}(x)=\{y \mid d(x, y) \leq R\}$.

Proof. Set $\tau=\tau(R, x)$ and $B=B_{r}(x)$. For $r \leq R$,

$$
\begin{aligned}
& \int_{B} \int_{B} p(t, y, z) \mu(d y) \mu(d z) \\
& \quad=\int_{B} \int_{B} p^{R, x}(t, y, z) \mu(d y) \mu(d z)+\int_{B} P_{y}\left(X_{t} \in B, \tau<t\right) \mu(d y) .
\end{aligned}
$$

Since $\left(\left\{X_{t}\right\}_{t>0},\left\{P_{x}\right\}_{x \in X}\right)$ is symmetric with respect to $\mu$,

$$
\begin{aligned}
& \int_{B} P_{y}\left(X_{t} \in B, t / 2<\tau<t\right) \mu(d y) \\
& =P_{\mu}\left(X_{0} \in B, X_{t} \in B, t / 2<\tau<t\right) \\
& \leq P_{\mu}\left(X_{0} \in B, X_{t} \in B, X_{s} \in \partial B_{R}(x) \text { for some } s \in[t / 2, t)\right) \\
& =P_{\mu}\left(X_{t} \in B, X_{0} \in B, X_{s} \in \partial B_{R}(x) \text { for some } s \in(0, t / 2]\right) \\
& =\int_{B} P_{y}\left(X_{t} \in B, \tau \leq t / 2\right) \mu(d y)
\end{aligned}
$$

Moreover,

$$
\begin{aligned}
P_{y}\left(X_{t} \in B, \tau \leq t / 2\right)=E_{y}\left(1_{\tau \leq t / 2} P_{X_{\tau}}\left(X_{t-\tau} \in B\right)\right) & \\
=E_{y}\left(1_{\tau \leq t / 2} \int_{B} p\left(t-\tau, y, X_{\tau}\right) \mu(d y)\right) & \\
& \leq \sup _{y \in B} P_{y}(\tau \leq t / 2) \sup _{\substack{y \in B, z \in \partial B_{R}(x) \\
t / 2 \leq s \leq t}} p(s, y, z) \mu(B) .
\end{aligned}
$$

Note that $p(s, y, z)^{2} \leq p(s, y, y) p(s, z, z)$. Also since $p(t, x, x)=\left\|p^{t / 2, x}\right\|_{2}^{2}$, where $p^{t, x}(y)=p(t, x, y), p(t, x, x)$ is monotonically decreasing. Therefore,

$$
P_{y}\left(X_{t} \in B, \tau \leq t / 2\right) \leq \sup _{y \in B} P_{y}(\tau \leq t / 2) \sup _{z \in D_{R}(x)} p(t / 2, z, z) \mu(B) .
$$


Combining this with (4.4) and (4.5), we obtain

$$
\begin{aligned}
\frac{1}{\mu(B)^{2}} \int_{B} \int_{B} p(t, y, z) & \mu(d y) \mu(d z) \\
\leq & \left\|T_{t}^{R, x}\right\|_{1 \rightarrow \infty}+2 \sup _{y \in B} P_{y}(\tau \leq t / 2) \sup _{z \in D_{R}(x)} p(t / 2, z, z) .
\end{aligned}
$$

Letting $r \rightarrow 0$, we obtain (4.3).

Proof of Theorem 2.9. First we show an diagonal estimate. Under the assumptions (A1) and (A2), Lemmas 4.3 and 4.4 holds with the same $r_{*}$. If necessary, we may replace $r_{*}$ by smaller value to make $r_{*} \omega \leq r_{1}$, where $\omega=$ $2^{1 / \beta} /\left(2^{1 / \beta}-1\right)$. Now fix $\delta>0$ and define $\rho(\delta)=\max \left\{b_{1}, b_{2} \delta^{\lambda / \beta}\right\}$ and $C(\delta)=$ $3 c \exp \left(-c^{\prime}(2 \delta)^{1 /(\beta-1)}\right)$. If $R^{\beta}=t \delta$ and $R \in\left(0, r_{*}\right]$, then Lemma 4.5 implies that, for any $x \in X$, there exists $x_{1} \in D_{R}(x)$ such that

$$
p(t, x, x) \leq \frac{\rho(\delta)}{h(R, x)}+C(\delta) p\left(t / 2, x_{1}, x_{1}\right) .
$$

Define $t_{n}=t / 2^{n}$ and $R_{n}=\left(t_{n} \delta\right)^{1 / \beta}$. Then there exists a sequence $\left\{x_{n}\right\}_{n \geq 0}$ such that $x_{n+1} \in D_{R_{n}}\left(x_{n}\right)$ and

$$
p\left(t_{n}, x_{n}, x_{n}\right) \leq \frac{\rho(\delta)}{h\left(R_{n}, x_{n}\right)}+C(\delta) p\left(t_{n+1}, x_{n+1}, x_{n+1}\right)
$$

for any $n \geq 0$. Therefore,

$$
p(t, x, x) \leq \rho(\delta) \sum_{i=0}^{n-1} \frac{C(\delta)^{i}}{h\left(R_{i}, x_{i}\right)}+C(\delta)^{n} p\left(t_{n}, x_{n}, x_{n}\right)
$$

for any $n \geq 0$. Since $x_{n} \in B_{R \omega}(x)$ for any $n$, Lemma 4.1 along with (H3) implies

$$
h\left(R_{n}, x_{n}\right) \geq c_{0}\left(\frac{R_{n}}{R \omega}\right)^{\lambda} h\left(R \omega, x_{n}\right) \geq \frac{c_{0} 2^{-n \lambda / \beta}}{M \omega^{\lambda}} h(R \omega, x) \geq\left(c_{*}\right)^{-1} G^{-n} h(R, x),
$$

where $c_{*}=M \omega^{\lambda} / c_{0}$ and $G=2^{\lambda / \beta}$. Combining this with Lemma 4.2, we see that there exists $b_{*}>0$ such that

$$
p(t, x, x) \leq \frac{\rho(\delta) c_{*}}{h(R, x)} \sum_{i=0}^{n-1}(C(\delta) G)^{i}+b_{*}(C(\delta) G)^{n}
$$

for any $n \geq 0$. Since $C(\delta) \rightarrow 0$ as $\delta \rightarrow+\infty$, we may choose $\delta \geq 1$ so that $C(\delta) G<1$. Then, there exists $a_{*}$ such that

$$
p(t, x, x) \leq \frac{a_{*}}{h\left((\delta t)^{1 / \beta}, x\right)} \leq \frac{a_{*}}{h\left(t^{1 / \beta}, x\right)}
$$

for any $x \in X$ and any $t \in\left(0, r_{*}^{\beta} / \delta\right]$. 
Next we show off-diagonal estimate. There is a well-established way of getting off-diagonal upper estimate from diagonal upper estimate. Here, we will adapt the discussion in [1]. Suppose that $x, y \in X$ with $d(x, y) \leq r_{*}$. Write $R=d(x, y)$. Define $A_{x}=\{z \mid d(x, z) \leq d(y, z)\}$ and $A_{y}=\{z \mid d(x, z) \geq d(y, z)\}$. Set $B_{1}=B_{r}(x)$ and $B_{2}=B_{r}(y)$. Then

$$
\begin{aligned}
& \int_{B_{1}} \int_{B_{2}} p(t, z, w) \mu(d z) \mu(d w) \leq \\
& \quad \int_{B_{1}}\left(P_{z}\left(X_{t} \in B_{2}, X_{t / 2} \in A_{x}\right)+P_{z}\left(X_{t} \in B_{2}, X_{t / 2} \in A_{y}\right)\right) \mu(d z) .
\end{aligned}
$$

If $r<R / 6, B_{R / 3}(z) \cap A_{y}=\emptyset$ for $z \in B_{1}$. Hence

$$
\begin{aligned}
P_{z}\left(X_{t} \in B_{2}, X_{t / 2} \in A_{y}\right) & \leq P_{z}\left(X_{t} \in B_{2}, \tau<t / 2\right) \\
& \leq E_{z}\left(1_{\tau<t / 2} \int_{B_{2}} p\left(t-\tau, X_{\tau}, w\right) \mu(d w)\right) \\
& \leq P_{z}(\tau \leq t / 2) \sup _{v \in D_{R / 2}(x) \cup B_{2}} p(t / 2, v, v) \mu\left(B_{2}\right) \\
& \leq C^{\prime}\left(R^{\beta} / t\right) \sup _{v \in D_{R / 2}(x) \cup B_{2}} p(t / 2, v, v) \mu\left(B_{2}\right),
\end{aligned}
$$

where $\tau=\tau(R / 3, z)$ and $C^{\prime}(s)=C\left(2 s / 3^{\beta}\right)$. Moreover, since the process is $\mu$-symmetric,

$$
\int_{B_{1}} P_{z}\left(X_{t} \in B_{2}, X_{t / 2} \in A_{x}\right) \mu(d z)=\int_{B_{2}} P_{w}\left(X_{t} \in B_{1}, X_{t / 2} \in A_{x}\right) \mu(d w) .
$$

Making use of those inequalities and letting $r \rightarrow 0$, we see that

$$
p(t, x, y) \leq 2 C^{\prime}\left(R^{\beta} / t\right) \sup _{z \in D_{R / 2}(x) \cup D_{R / 2}(y)} p(t / 2, z, z) .
$$

Let $z \in D_{R / 2}(x) \cup D_{R / 2}(y)$. Then $d(x, z) \leq 3 R / 2$. If $(t / 2)^{1 / \beta} \geq 3 R / 2$, then Lemma 4.1 and (H3) imply

$$
h\left((t / 2)^{1 / \beta}, z\right) \geq M^{-1} h\left((t / 2)^{1 / \beta}, x\right) \geq c_{0} M^{-1} 2^{-\lambda / \beta} h\left(t^{1 / \beta}, x\right) .
$$

If $(t / 2)^{1 / \beta}<3 R / 2$, then

$$
\begin{aligned}
h\left((t / 2)^{1 / \beta}, z\right) & \geq c_{0} 2^{-\lambda / \beta}(2 / 3)^{\lambda}\left(\frac{R^{\beta}}{t}\right)^{-\lambda / \beta} h(3 R / 2, z) \\
& \geq c_{0} 2^{-\lambda / \beta}(2 / 3)^{\lambda} M^{-1}\left(\frac{R^{\beta}}{t}\right)^{-\lambda / \beta} h(3 R / 2, x) \\
& \geq c_{0} 2^{-\lambda / \beta}(2 / 3)^{\lambda} M^{-1}\left(\frac{R^{\beta}}{t}\right)^{-\lambda / \beta} h\left((t / 2)^{1 / \beta}, x\right) \\
& \geq\left(c_{0}\right)^{2} 2^{-2 \lambda / \beta}(2 / 3)^{\lambda} M^{-1}\left(\frac{R^{\beta}}{t}\right)^{-\lambda / \beta} h\left(t^{1 / \beta}, x\right)
\end{aligned}
$$


Hence, there exists $B>0$ such that

$$
\frac{1}{h\left((t / 2)^{1 / \beta}, z\right)} \leq B \max \left\{1,\left(\frac{R^{\beta}}{t}\right)^{\lambda / \beta}\right\} \frac{1}{h\left(t^{1 / \beta}, x\right)} .
$$

Therefore, by (4.6) and (4.7),

$$
p(t, x, y) \leq 2 a_{*} B \max \left\{1,\left(\frac{R^{\beta}}{t}\right)^{\lambda / \beta}\right\} \frac{1}{h\left(t^{1 / \beta}, x\right)} C^{\prime}\left(R^{\beta} / t\right) .
$$

Since there exists $c^{\prime \prime}$ and $c^{\prime \prime \prime}$ such that

$$
\max \left\{1, \delta^{\lambda / \beta}\right\} C^{\prime}(\delta) \leq c^{\prime \prime} \exp \left(-c^{\prime \prime \prime} \delta^{1 /(\beta-1)}\right)
$$

for any $\delta>0$, we obtain $(2.8)$

Next we prove Theorem 2.10 by adapting the arguments in the proof of $[1$, Lemma 3.9].

Lemma 4.6. Suppose that (2.9) holds for any $t \in\left(0, t_{*}\right]$ and any $x, y \in X$.

(1) There exists $c$ and $c^{\prime}$ such that

$$
P_{x}\left(d\left(x, X_{t}\right)>r\right) \leq c \exp \left(-c^{\prime}\left(\frac{r^{\beta}}{t}\right)^{\frac{1}{\beta-1}}\right)
$$

for any $x \in X$, any $t \in\left(0, t_{*}\right]$ and any $r>0$.

(2) There exist positive constants $c$ and $c^{\prime}$ such that (4.2) holds for any $x \in X$, any $t \in\left(0, t_{*}\right]$ and any $r>0$.

Proof. (1) Choose $\theta>1$ and $\alpha>1$ such that $V(\theta r, x) \leq \alpha V(r, x)$ for any $x \in X$ and any $r>0$. Let $\theta=\alpha^{\delta}$. Then

$$
\begin{aligned}
P_{x}\left(d\left(x, X_{t}\right)>r\right) & =\sum_{n=0}^{+\infty} \int_{B_{\theta^{n+1}}(x) \backslash B_{\theta^{n}}(x)} p(t, x, y) \mu(d y) \\
& \leq c_{1} \sum_{n=0}^{+\infty} \alpha^{n+1} \frac{V(r, x)}{V\left(t^{1 / \beta}, x\right)} \exp \left(-c_{2}\left(\frac{\left(\theta^{n} r\right)^{\beta}}{t}\right)^{\frac{1}{\beta-1}}\right) \\
& =c_{1} \alpha \frac{V(r, x)}{V\left(t^{1 / \beta}, x\right)} \sum_{n=0}^{+\infty} \alpha^{n} \exp \left(-c_{2}\left(\frac{r^{\beta}}{t}\right)^{\frac{1}{\beta-1}} \alpha^{n \frac{\beta \delta}{\beta-1}}\right) \\
& \leq c_{1} \frac{\alpha}{\alpha-1} \frac{V(r, x)}{V\left(t^{1 / \beta}, x\right)} \int_{1}^{+\infty} \exp \left(-c_{2}\left(\frac{r^{\beta}}{\theta^{\beta} t}\right)^{\frac{1}{\beta-1}} x^{\frac{\beta \delta}{\beta-1}}\right) d x
\end{aligned}
$$

Suppose $r^{\beta} \geq t$. Then by Lemma $4.1, V(r, x) / V\left(t^{1 / \beta}, x\right) \leq c_{3}\left(r^{\beta} / t\right)^{\rho}$. Therefore, by [1, Lemma 3.7], there exist $c_{4}>0$ and $c_{5}>0$ such that

$$
P_{x}\left(d\left(x, X_{t}\right)>r\right) \leq c_{4}\left(\frac{r^{\beta}}{t}\right)^{\rho-\frac{1}{\beta-1}} \exp \left(-c_{5}\left(\frac{r^{\beta}}{t}\right)^{\frac{1}{\beta-1}}\right)
$$


Choosing suitable constants $c$ and $c^{\prime}$, we immediately have (4.8) when $r^{\beta} \geq t$. Note that we may choose $c$ and $c^{\prime}$ so that $1 \leq c e^{-c^{\prime}}$. Hence (4.8) holds for $t>r^{\beta}$ as well.

(2) By the discussion in the proof of [1, Lemma 3.9], we see that

$$
P_{x}(\tau(x, r)<t) \leq 2 \sup _{s<t} \sup _{y \in X} P_{y}\left(d\left(y, X_{s}\right)>r / 2\right) .
$$

Now (1) immediately implies (2).

Lemma 4.7. If $\mu$ has the volume doubling property on $\left(0, r_{*}\right]$, then there exists $\rho \in(0,1)$ such that $V(r / 2, x) \leq \rho V(r, x)$ for any $r \leq \min \left\{r_{*}, \operatorname{diam}(X) / 3\right\}$ and any $x \in X$.

Proof. Let $r \leq \min \left\{r_{*}, \operatorname{diam}(X) / 2\right\}$ and let $x \in X$. Then there exists $z \in X$ such that $d(x, z) \geq r$. Since $(X, d)$ is connected, we may choose $y \in X$ with $d(x, y)=3 r / 4$. Note that $B_{r / 2}(x) \cap B_{r / 4}(y)=\emptyset$ and $B_{r / 2}(x) \cup B_{r / 4}(x) \subset$ $B_{r}(x)$. Now Since $B_{r / 2}(x) \subseteq B_{5 r / 4}(y)$, the volume doubling property implies $V(r / 2, x) \leq V(5 r / 4, y) \leq c V(r / 4, y)$, where $c>0$ is independent of $r, x$ and $y$. Hence $V(r, x) \geq\left(1+c^{-1}\right) V(r / 2, x)$.

Proof of Theorem 2.10. Let $c_{*} \geq 1$. Set $r_{*}=\left(c_{*}\right)^{-1} \min \left\{\left(t_{*}\right)^{1 / \beta}, \operatorname{diam}(X)\right)$. Let $r \in\left(0, r_{*}\right]$. Then, by $(\mathrm{H} 3)$,

$$
\begin{aligned}
& P_{y}\left(\tau(x, r)>\left(c_{*} r\right)^{\beta}\right) \leq P_{y}\left(X_{\left(c_{*} r\right)^{\beta}} \in B_{r}(x)\right) \leq \int_{B_{r}(x)} p\left(\left(c_{*} r\right)^{\beta}, y, z\right) \mu(d z) \\
& \quad \leq c_{1} \int_{B_{r}(x)} \frac{1}{V\left(c_{*} r, z\right)} \mu(d z) \leq c_{1} \int_{B_{r}(x)} \frac{M}{V\left(c_{*} r, x\right)} \mu(d z)=c_{1} M \frac{V(r, x)}{V\left(c_{*} r, x\right)}
\end{aligned}
$$

By Lemma 4.7, we may choose $c_{*}$ so that the last value of the above inequality is less than $1 / 2$. The Markov property of the process implies that

$$
P_{y}\left(\tau(x, r)>k\left(c_{*} r\right)^{\beta}\right) \leq 2^{-k} .
$$

Hence, if $R=\left(c_{*} r\right)^{\beta}$,

$$
E_{x}(\tau(x, r)) \leq \sum_{k \geq 0} P_{y}((k+1) R \geq \tau(x, r)>k R)(k+1) R \leq 4 c_{*}^{\beta} r^{\beta}
$$

for any $r \in\left(0, r_{*}\right]$.

To obtain the lower bound, choose $a>0$ satisfying $c \exp \left(-c^{\prime \prime} a^{1 /(\beta-1)}\right)=$ $1 / 2$. If $r \leq\left(a t_{*}\right)^{1 / b}$, then Lemma 4.6 shows that $P_{x}\left(\tau(x, r) \leq r^{\beta} / a\right) \leq 1 / 2$. Since $(\mathcal{E}, \mathcal{F})$ is convervative, $P_{x}\left(X_{t} \in X\right)=1$. Therefore,

$$
E_{x}(\tau(x, r)) \geq P_{x}\left(\tau(x, r)>r^{\beta} / a\right) r^{\beta} / a \geq \frac{r^{\beta}}{2 a}
$$


Finally we prove Theorem 2.13. As we mentioned, the proof here depends on a modification of known techniques developed for the homogeneous case. See $[1,3,8,11]$ for example.

Proof of Theorem 2.13. First we give a proof of diagonal estimate. By the exit time estimate, we have Lemma 4.4. Set $\delta=r^{\beta} / t$, where $r \in\left(0, r_{*}\right]$. Then by (4.2), if $\delta$ is sufficiently large, then $P_{x}(\tau(r, x) \leq t / 2) \leq 1 / 2$. Since $(\mathcal{E}, \mathcal{F})$ is conservative,

$$
\begin{aligned}
1 / 2 \leq 1-P_{x}(\tau(r, x) \leq & t / 2) \leq P_{x}\left(X_{t / 2} \in B_{r}(x)\right) \\
& =\int_{B_{r}(x)} p(t / 2, x, y) \mu(d y) \leq \sqrt{\mu\left(B_{r}(x)\right)} \sqrt{p(t, x, x)} .
\end{aligned}
$$

Using the volume doubling property, we see that

$$
\frac{c}{V\left(t^{1 / \beta}, x\right)} \leq p(t, x, x)
$$

for any $t \in\left(0, t_{*}\right]$, where $t_{*}=\left(r_{*}\right)^{\beta} / \delta$. To obtain off-diagonal estimate, we use the classical chaining argument. (See [1] for example.) Note that

$$
p(t, x, y)=\int_{X^{n-1}} p\left(\frac{t}{n}, x, z_{1}\right) p\left(\frac{t}{n}, z_{1}, z_{2}\right) \cdots p\left(\frac{t}{n}, z_{n-1}, y\right) \mu\left(d z_{1}\right) \cdots \mu\left(d z_{n-1}\right) .
$$

Now fix $x$ and $y$. We may assume that $\epsilon \leq 3$ without loss of generality. Write $R=d(x, y)$. If $R \leq \epsilon t^{1 / \beta}$, then the near diagonal estimate implies (2.12). Therefore, we suppose that $R>\epsilon t^{1 / \beta}$. Since $(3 R / \epsilon)^{\beta} / t \geq 1$, there exists $n \in \mathbb{N}$ such that

$$
\left(\frac{3}{\epsilon}\right)^{\beta} \frac{R^{\beta}}{t} \leq n^{\beta-1} \leq 2^{\beta-1}\left(\frac{3}{\epsilon}\right)^{\beta} \frac{R^{\beta}}{t} .
$$

Using the midpoint property, we may choose a sequence $\left\{x_{i}\right\}_{i=0}^{n}$ such that $x_{0}=$ $x, x_{n}=y$ and $d\left(x_{i}, x_{i+1}\right)=R / n$ for any $i=0,1, \ldots, n-1$. Define $B_{i}=$ $B_{R / n}\left(x_{i}\right)$ for $i=1, \ldots, n-1$. If $z_{i} \in B_{i}$ and $z_{i+1} \in B_{i+1}$, then $d\left(z_{i}, z_{i+1}\right) \leq$ $3 R / n$. Since $3 R / n \leq \epsilon(t / n)^{1 / \beta}$ and $R / n \leq(t / n)^{1 / \beta}$, the near diagonal estimate and (H3) yields

$$
p\left(t, z_{i}, z_{i+1}\right) \geq \frac{c}{V\left((t / n)^{1 / \beta}, z_{i}\right)} \geq \frac{c}{M V\left((t / n)^{1 / \beta}, x_{i}\right)} .
$$

Hence

$$
\begin{aligned}
& p(t, x, y) \\
& \geq \int_{B_{1} \times \ldots \times B_{n-1}} p\left(\frac{t}{n}, x, z_{1}\right) p\left(\frac{t}{n}, z_{1}, z_{2}\right) \cdots p\left(\frac{t}{n}, z_{n-1}, y\right) \mu\left(d z_{1}\right) \cdots \mu\left(d z_{n-1}\right) \\
& \geq(c / M)^{n} \frac{1}{V\left((t / n)^{1 / \beta}, x\right)} \prod_{i=1}^{n-1} \frac{V\left(R / n, x_{i}\right)}{V\left((t / n)^{1 / \beta}, x_{i}\right)} \\
& \geq(c / M)^{n} \frac{1}{V\left(t^{1 / \beta}, x\right)} \prod_{i=1}^{n-1} \frac{V\left(R / n, x_{i}\right)}{V\left((t / n)^{1 / \beta}, x_{i}\right)}
\end{aligned}
$$


By Lemma 4.1 and (4.10),

$$
V\left(R / n, x_{i}\right) / V\left((t / n)^{1 / \beta}, x_{i}\right) \geq c_{o}\left(\frac{R^{\beta}}{t n^{\beta-1}}\right)^{\lambda / \beta} \geq c_{0} 2^{-\lambda(\beta-1) / \beta}(\epsilon / 3)^{\lambda} .
$$

Therefore there exists $L>1$ such that

$$
p(t, x, y) \geq \frac{L^{-n}}{V\left(t^{1 / \beta}, x\right)} .
$$

Now the desired estimate follows immediately from (4.10).

\section{Example}

In this section, we will apply our main results to a time change of the Brownian motion on $[0,1]$ reflecting at the boundary with respect to a self-similar measure. For simplicity, we will only deal with self-similar measures with three weight $\left(\mu_{1}, \mu_{2}, \mu_{2}\right)$. See subsequent paper [16] for general cases.

Let $K=[0,1]$. Define $F_{i}(x)=\left(x-a_{i}\right) / 3+a_{i}$ for $i=1,2,3$, where $a_{1}=$ $0, a_{2}=1 / 2$ and $a_{3}=1$. Then $K=F_{1}(K) \cup F_{2}(K) \cup F_{3}(K)$. Define $\Sigma=$ $\{1,2,3\}^{\mathbb{N}}, W_{m}=\{1,2,3\}^{m}$ and $W_{*}=\cup_{m \geq 0}$, where $W_{0}=\{\emptyset\}$. For $w \in W_{*}$, $|w|$ is the length of $|w|$, i.e. $w \in W_{|w|}$. We define $F_{w}=F_{w_{1}} \circ \ldots \circ F_{w_{m}}$ for $w=w_{1} \ldots w_{m} \in W_{*}$. Also $K_{w}=F_{w}(K)$. It is well known that there exists a continuous surjection $\pi: \Sigma \rightarrow K$ such that $\pi(\omega)=\cap_{m \geq 0} K_{\omega_{1} \ldots \omega_{m}}$ for any $\omega=\omega_{1} \omega_{2} \ldots$. Note that $\pi\left(2(1)^{\infty}\right)=\pi\left(1(3)^{\infty}\right)=1 / 3$ and $\pi\left(3(1)^{\infty}\right)=\pi\left(2(3)^{\infty}\right)$, where $(w)^{\infty}=w w w \ldots$ for $w \in W_{*}$.

Proposition 5.1. If $\pi(\omega)=\pi(\eta)$ for $\omega, \eta \in \Sigma$ with $\omega \neq \eta$, then there exists $w \in W_{*}$ such that $\{\omega, \eta\}$ is $\left\{w 2(1)^{\infty}, w 1(3)^{\infty}\right\}$ or $\left\{w 3(1)^{\infty}, w 2(3)^{\infty}\right\}$.

Now we define $\mathcal{E}(u, v)=\int_{0}^{1} u^{\prime}(x) v^{\prime}(x) d x$ for $u, v \in C^{1}([0,1])$. Let $\mathcal{E}_{1}(u, v)=$ $\mathcal{E}(u, v)+\int_{0}^{1} u(x) v(x) d x . \mathcal{F}$ is the completion of $C^{1}([0,1])$ with respect to the inner product $\mathcal{E}_{1}$. Then $\mathcal{F} \subset C([0,1])$ and, in fact,

$$
|u(x)-u(y)|^{2} \leq \mathcal{E}(u, u)|x-y|
$$

Also $(\mathcal{E}, \mathcal{F})$ has a self-similarity: for any $u \in \mathcal{F}, u \circ F_{i} \in \mathcal{F}$ for $i=1,2,3$ and

$$
\mathcal{E}(u, u)=\sum_{i=1}^{3} 3 \mathcal{E}\left(u \circ F_{i}, u \circ F_{i}\right) .
$$

$(\mathcal{E}, \mathcal{F})$ is a local regular Dirichlet form on $L^{2}(K, d x)$ and the associated diffusion process is the Brownian motion on $[0,1]$.

Proposition 5.2. Let $\mu$ be a self-similar measure on $K$ with weight $\left(\mu_{1}, \mu_{2}, \mu_{3}\right) \in$ $(0,1)^{3}: \mu_{1}+\mu_{2}+\mu_{3}=1, \mu$ is a Borel regular measure on $K$ and $\mu\left(K_{w}\right)=\mu_{w}$ for any $w \in W_{*}$, where $\mu_{w}=\mu_{w_{1}} \ldots \mu_{w_{m}}$ for $w=w_{1} \ldots w_{m}$. Then $(\mathcal{E}, \mathcal{F})$ is a local regular Dirichlet form on $L^{2}(K, \mu)$. 
The diffusion process associated with $(\mathcal{E}, \mathcal{F})$ on $L^{2}(K, \mu)$ is a time change of the Brownian motion on $[0,1]$.

Next we define a distance associated with $(\mathcal{E}, \mathcal{F})$ on $L^{2}(K, \mu)$. Let $d_{S}$ be the unique positive number which satisfies

$$
\left(\mu_{1} / 3\right)^{d_{S} / 2}+\left(\mu_{2} / 3\right)^{d_{S} / 2}+\left(\mu_{3} / 3\right)^{d_{S} / 2}=1 .
$$

$d_{S}$ is called the spectral exponent and gives an asymptotic order of the distribution of eigenvalues of the Laplacian associated with $(\mathcal{E}, \mathcal{F})$ on $L^{2}(K, \mu)$. See [18] for details. Define $p_{i}=\left(\mu_{i} / 3\right)^{d_{S} / 2}$ for $i=1,2,3$. For $x, y \in K$ with $x \leq y$, set $d_{m}(x, y)=\sum_{w \in W_{m}(x, y)} p_{w}$, where $W_{m}(x, y)=\left\{w \mid w \in W_{m}, K_{w} \subseteq[x, y]\right\}$. Define $d(x, y)=\lim _{m \geq \infty} d_{m}(x, y)$. Then, $d(x, y)$ is a distance on $K$, which possesses the midpoint property.

Theorem 5.3. Let $p(t, x, y)$ be the heat kernel associated with the Dirichlet form $(\mathcal{E}, \mathcal{F})$ on $L^{2}(K, \mu)$. If $\mu_{1}=\mu_{3}$, then there exist positive constants $c_{1}, c_{2}, c_{3}$ and $c_{4}$ such that

$$
\begin{aligned}
\frac{c_{1}}{V\left(t^{1 / \beta}, x\right)} \exp \left(-c_{2}\left(\frac{d(x, y)^{\beta}}{t}\right)^{\frac{1}{\beta-1}}\right) & \\
p(t, x, y) & \leq \frac{c_{3}}{V\left(t^{1 / \beta}, x\right)} \exp \left(-c_{4}\left(\frac{d(x, y)^{\beta}}{t}\right)^{\frac{1}{\beta-1}}\right)
\end{aligned}
$$

for any $x, y \in K$ and any $t \in(0,1]$, where $\beta=2 / d_{S}$ and $V(r, x)=\mu\left(B_{r}(x)\right)$.

Unless $\mu_{1}=\mu_{2}=\mu_{3}$, the local dimension of $\mu, d_{L}(x)=\varlimsup_{r \rightarrow 0} \log V(r, x) / \log r$ is quite sensitive to $x$ and has multifractal structure. More precisely, define $A_{\alpha}=\left\{x \mid x \in[0,1], d_{L}(x)=\alpha\right\}$ and let $f(\alpha)$ be the Hausdorff dimension of $A_{\alpha}$ with respect to the distance $d$. Then $f(\alpha)$ is non-trivial continuous convex function. See [13] for details.

The rest of this section is devoted to the proof of Theorem 5.3.

Lemma 5.4. Suppose $\mu_{1}=\mu_{3}$. Define

$$
\Lambda_{s}=\left\{w \mid w \in W_{*}, p_{w_{1} \ldots w_{m-1}}>s \geq p_{w}\right\} .
$$

for $s \in(0,1]$. Then there exists $M>0$ such that $|w|-|v| \leq M$ and $\mu\left(K_{w}\right) \leq$ $M \mu\left(K_{v}\right)$ for any $w, v \in \Lambda_{s}$ with $K_{w} \cap K_{v} \neq \emptyset$ and any $s \in(0,1]$.

Proof. If $K_{w} \cap K_{v} \neq \emptyset$ for $w, v \in \Lambda_{s}$ and $w \neq v$, then, by Proposition 5.1, we may suppose $w=\omega_{1} \ldots \omega_{m}$ and $v=\eta_{1} \ldots \eta_{n}$, where $\omega=\operatorname{ai}(1)^{\infty}$ and $\eta=a j(3)^{\infty}$ for some $a \in W_{*}$ and some $(i, j)=(2,1),(3,2)$. Let $C=\min \left\{p_{1}, p_{2}, p_{3}\right\}$. Then $C p_{v} \leq p_{w} \leq C^{-1} p_{v}$. Since $p_{1}=p_{3}$, the boundedness of $p_{w} / p_{v}$ implies the boundedness of $|w|-|v|$. Note that $p_{w}=\left(\mu_{w} / 3^{|w|}\right)^{d_{S} / 2}$ and $p_{v}=\left(\mu_{v} / 3^{|v|}\right)^{d_{S} / 2}$. Hence the boundedness of $|w|-|v|$ implies the boundedness of $\mu_{w} / \mu_{v}$.

For $x \in K$, define $\Lambda_{s, x}=\left\{w \mid w \in \Lambda_{s}, x \in K_{w}\right\}, K_{s}(x)=\cup_{w \in \Lambda_{s, x}} K_{w}$, $\Lambda_{s, x}^{1}=\left\{w \mid w \in \Lambda_{s}, K_{w} \cap K_{v} \neq \emptyset\right.$ for some $\left.v \in \Lambda_{s, x}\right\}$ and $U_{s}(x)=\cup_{w \in \Lambda_{s, x}^{1}} K_{w}$. Then,

$$
B_{C s}(x) \subseteq U_{s}(x) \subseteq B_{3 s}(x)
$$


for any $x \in K$ and any $s \in(0,1]$. Note that $\# \Lambda_{s, x} \leq 2$ and $\# \Lambda_{s, x}^{1} \leq 4$, where \# is the number of elements. Hence by Lemma 5.4, there exist positive constants $A_{1}$ and $A_{2}$ such that

$$
A_{1} \mu_{w} \leq \mu\left(U_{s}(x)\right) \leq A_{2} \mu_{w}
$$

for any $x \in K, s \in(0,1]$ and any $w \in \Lambda_{s, x}$.

Proof of Theorem 5.3. To use Theorems 2.9 and 2.13, we need to show the volume doubling property, the local Nash inequality, the exit time estimate and the near diagonal lower estimate.

Volume doubling property: Choose $\max \left\{p_{1}, p_{2}, p_{3}\right\}<\alpha<1$. Let $x=\pi\left(\omega_{1} \omega_{2} \ldots\right)$. Then $w=\omega_{1} \ldots \omega_{m} \in \Lambda_{s, x}$ for some $m$. We see that $w^{\prime}=\omega_{1} \ldots \omega_{m+1} \in \Lambda_{\alpha s, x}$. Hence by (5.4), $\mu\left(U_{s}(x)\right) \geq \mu_{w} \geq \mu_{w^{\prime}} / B \geq\left(A_{2} B\right)^{-1} \mu\left(U_{\alpha s}(x)\right)$, where $B=$ $\max \left\{\mu_{1}, \mu_{2}\right\}$. Using (5.3), we verify the volume doubling property.

Local Nash inequality: By integrating (5.1) with respect to $\mu(d x)$ and $\mu(d y)$, there exist $c>0$ such that

$$
\mathcal{E}(u, u) \geq c \int_{K}(u(x)-\bar{u})^{2} \mu(d x),
$$

for any $u \in \mathcal{F}$, where $\bar{u}=\int_{K} u(x) \mu(d x)$. Define $\Lambda_{s}(u)=\left\{w \mid w \in \Lambda_{s}, K_{w} \cap\right.$ $\operatorname{supp}(u) \neq \emptyset\}$ for any $u \in \mathcal{F}$. Set $u_{w}=u \circ F_{w}$ Then, by the self-similarity of $(\mathcal{E}, \mathcal{F})$,

$$
\begin{aligned}
\mathcal{E}(u, u) & =\sum_{w \in \Lambda_{s}(u)} 3^{|w|} \mathcal{E}\left(u_{w}, u_{w}\right) \geq c \sum_{w \in \Lambda_{s}(u)} 3^{|w|} \int_{K}\left(u_{w}-\overline{u_{w}}\right)^{2} \mu(d x) \\
& =c \sum_{w \in \Lambda_{s}(u)}\left(p_{w}\right)^{-\beta}\left(\int_{K_{w}} u(x)^{2} \mu(d x)-\left(\mu_{w}\right)^{-1}\left(\int_{K_{w}} u(x) \mu(d x)\right)^{2}\right) \\
& \geq \frac{c\|u\|_{2}^{2}}{s^{\beta}}-\frac{c\|u\|_{1}^{2}}{s^{\beta} \inf _{w \in \Lambda_{s}(u)} \mu_{w}}
\end{aligned}
$$

Using (5.3), (5.4) and the volume doubling property, we also obtain that there exists $a>0$ such that $\inf _{w \in \Lambda_{s}(u)} \mu_{w} \geq a \inf _{x \in \operatorname{supp}(u)} V(s, x)$. Thus (5.6) implies the local Nash inequality.

Exit time estimate: By [15, Appendix B],

$$
E_{x}\left(\tau_{U_{s}(x)}\right)=R\left(x, \partial U_{s}(x)\right) \int_{K} \varphi_{x, s}(y) \mu(d y),
$$

where $R\left(x, \partial U_{s}(x)\right)$ is the effective resistance between $x$ and $\partial U_{s}(x)$ and $\varphi_{x, s}$ is a piecewise linear function satisfying $\varphi_{x, s}(x)=1$ and $\operatorname{supp}\left(\varphi_{x, s}\right)=U_{s}(x)$. If $U_{s}(x)=[x(s), y(s)]$, then $R\left(x, \partial U_{s}(x)\right)=|x-x(s)||y(s)-x| /|y(s)-x(s)|$. Set $\Gamma=\left\{(x, s, w) \mid x \in K, s \in(0,1], w \in \Lambda_{s, x}\right\}$. By Lemma 5.4, there exist positive constants $b_{1}$ and $b_{2}$ such that $b_{1} 3^{-|w|} \leq|x-x(s)|,|y-y(s)| \leq b_{2} 3^{-|w|}$ for any $(x, s, w) \in \Gamma$. Hence

$$
0<\inf _{(x, s, w) \in \Gamma} R\left(x, \partial U_{s}(x)\right) 3^{|w|} \leq \sup _{(x, s, w) \in \Gamma} R\left(x, \partial U_{s}(x)\right) 3^{|w|}<+\infty
$$


Also by Lemma 5.4, $\inf _{(x, s, w) \in \Gamma}\left(\min _{y \in K_{w}} \varphi_{s, x}(y)\right)>0$. Therefore, there exists $b_{5}$ and $b_{6}$ such that $b_{5} \mu_{w} \leq \int_{K} \varphi_{x, s}(y) \mu(d y) \leq b_{6} \mu_{w}$ for any $(x, s, w) \in \Gamma$. As a consequence, $s^{-b} E_{x}\left(\tau_{U_{s}(x)}\right)$ is uniformly bonded from above and below with respect to $(x, s) \in K \times(0,1]$. Using (5.3), we deduce (2.7).

Near diagonal estimate: Note that we already have diagonal lower estimate $\overline{c V\left(t^{1 / \beta}, x\right)^{-1} \leq p(t, x, x)}$. By the discussions in the proofs of [8, Lemma 6.4] and [14, Lemma 5.2], $|p(t, x, y)-p(t, x, z)|^{2} \leq|y-z| p(t, x, x) t^{-1}$. Therefore,

$$
\begin{aligned}
p(t, x, y) \geq p(t, x, x)-|p(t, x, y)-p(t, x, x)| & \\
& \geq p(t, x, x)\left(1-c \frac{|x-y|^{1 / 2} V\left(t^{1 / \beta}, x\right)^{1 / 2}}{t^{1 / 2}}\right) .
\end{aligned}
$$

Let $x=\pi\left(\omega_{1} \omega_{2} \ldots\right)$. Define $\omega(k)=\omega_{1} \ldots \omega_{k}$ for any $k \in \mathbb{N}$. Let $R=t^{1 / \beta}$. Then $\omega(m) \in \Lambda_{R, x}$ for some $m$. By the volume doubling property and (5.4), there exists $a_{1}>0$ such that $V(R, x) \leq a_{1} \mu_{\omega(m)}$. Also, there exists $a_{2}>0$ such that $t=R^{\beta} \geq \mu_{\omega(m)} / 3^{m}$. Set $\alpha_{0}=\min \left\{p_{1}, p_{2}, p_{3}\right\}$. Then, for any $n \geq 1$, $\omega(m+k) \in \Lambda_{\left(a_{0}\right)^{n} R, x}$ for some $k \geq n$. Therefore there exists $a_{3}>0$ such that $|x-y| \leq a_{3} 3^{-(m+n)}$ for any $y \in U_{\left(\alpha_{0}\right)^{n} R}(x)$. Combining those inequalities, we see

$$
\frac{|x-y| V\left(t^{1 / \beta}, x\right)}{t} \leq a_{1} a_{3}\left(a_{2}\right)^{-1} 3^{-n}
$$

for any $y \in U_{\left(\alpha_{0}\right)^{n} R}(x)$. Therefore, by (5.7), if $\epsilon$ is sufficiently small, $p(t, x, y) \geq$ $p(t, x, x) / 2$ for any $y \in B_{\epsilon t^{1 / \beta}}(x)$. This immediately imply the near diagonal lower estimate.

\section{References}

[1] M. T. Barlow, Diffusion on fractals, Lecture notes Math. vol. 1690, Springer, 1998.

[2] M. T. Barlow and R. F. Bass, Brownian motion and harmonic analysis on Sierpinski carpets, Canad. J. Math. 51 (1999), 673-744.

[3] M. T. Barlow and T. Kumagai, Transition density asymptotics for some diffusion processes with multi-fractal structures, Electron. J. Probab. 6 (2001).

[4] M. T. Barlow and E. A. Perkins, Brownian motion on the Sierpinski gasket, Probab. Theory Related Fields 79 (1988), 542-624.

[5] E. Carlen, S. Kusuoka, and D. Stroock, Upper bounds for symmetric Markov transition functions, Ann. Inst. Henri Poincaré 23 (1987), 245287.

[6] T. Coulhon, Ultracontractivity and Nash type inequalities, J. Functional Analysis 141 (1996), 510-539. 
[7] E. B. Davies, Heat Kernels and Spectral Theory, Cambridge Tracts in Math. vol 92, Cambridge University Press, 1989.

[8] P. J. Fitzsimmons, B. M. Hambly, and T. Kumagai, Transition density estimates for Brownian motion on affine nested fractals, Comm. Math. Phys. 165 (1994), 595-620.

[9] M. Fukushima, Y. Oshima, and M. Takeda, Dirichlet Forms and Symmetric Markov Processes, de Gruyter Studies in Math. vol. 19, de Gruyter, Berlin, 1994.

[10] A. Grigor'yan, The heat equation on noncompact Riemannian manifolds. (in Russian), Mat. Sb. 182 (1991), 55-87, English translation in Math. USSR-Sb. 72(1992), 47-77.

[11] A. Grigor'yan and A. Telcs, Sub-Gaussian estimates of heat kernels on infinite graphs, Duke Math. J. 109 (2001), $451-510$.

[12] A. Grigor'yan and A. Telcs, Harnack inequalities and sub-Gaussian estimates for random walks, Math. Ann. 324 (2002), 521-556.

[13] B. M. Hambly, J. Kigami, and T. Kumagai, Multifractal formalisms for the local spectral and walk dimensions, Math. Proc. Cambridge Phil. Soc. 132 (2002), $555-571$.

[14] B. M. Hambly and T. Kumagai, Transition density estimates for diffusion processes on post critically finite self-similar fractals, Proc. London Math. Soc. (3) 78 (1999), 431-458.

[15] J. Kigami, Harmonic analysis for resistance forms, J. Functional Analysis 204 (2003), 399-444.

[16] J. Kigami, Volume doubling measures and heat kernel estimates on selfsimilar sets, in preparation.

[17] J. Kigami, Analysis on Fractals, Cambridge Tracts in Math. vol. 143, Cambridge Univresity Press, 2001.

[18] J. Kigami and M. L. Lapidus, Weyl's problem for the spectral distribution of Laplacians on p.c.f. self-similar fractals, Comm. Math. Phys. 158 (1993), 93-125.

[19] T. Kumagai, Estimates of the transition densities for Brownian motion on nested fractals, Probab. Theory Related Fields 96 (1993), 205-224.

[20] P. Li and S.-T. Yau, On the parabolic kernel of the Schrödinger operator, Acta Math. 156 (1986), $153-201$.

[21] J. Nash, Continuity of solutions of parabolic and elliptic equations, Amer. J. Math. 80 (1958), 931-954. 
[22] L. Saloff-Coste, A note on Poincaré, Sobolev, and Harnack inequalities, Duke Math. J. 65 no. 3, Internat. Math. Res. Notices (1992), 27-38.

[23] A. Telcs, Volume and time doubling of graphs and random walks: the strongly recurrent case, Comm. Pure Appl. Maht. 54 (2001), 976 - 1018.

[24] A. Telcs, Random walks on graphs with volume and time doubling, preprint.

[25] M. Tomisaki, Comparison theorems on Dirichlet norms and their applications, Forum Math. 2 (1990), 277-295. 